\title{
Prevalence and treatment of small intestinal bacterial overgrowth in postoperative patients with colorectal cancer
}

\author{
LIHUA DENG $^{1}$, YANG LIU ${ }^{1}$, DONGSHENG ZHANG ${ }^{2}$, YUAN LI $^{1}$ and LIN XU ${ }^{1}$ \\ Departments of ${ }^{1}$ Gastroenterology and ${ }^{2}$ Hepatobiliary Surgery, Qingdao Municipal Hospital \\ Affiliated to Qingdao University, Qingdao, Shandong 266071, P.R. China
}

Received July 21, 2015; Accepted February 25, 2016

DOI: $10.3892 / \mathrm{mco} .2016 .807$

\begin{abstract}
To investigate the prevalence of small intestinal bacterial overgrowth (SIBO) in patients with colorectal cancer (CRC) after surgical treatment and observe whether gastrointestinal symptoms may improve with rifaximin, 43 postoperative $\mathrm{CRC}$ patients (CRC group) and 30 healthy individuals (normal group) were subjected to the glucose hydrogen breath test (GHBT). All the patients were asked to evaluate the gastrointestinal symptoms using the visual analogue scale (VAS). SIBO-positive patients were administered rifaximin for 10 days on the basis of the original treatment. After the treatment, the patients were asked to undergo GHBT and re-evaluate the gastrointestinal symptoms score (GISS). The results demonstrated that 18 of the 43 postoperative patients with CRC were SIBO-positive (41.86\%), which was significantly higher compared with the incidence in normal controls $(6.67 \%)(\mathrm{P}<0.05)$. GISS was higher in SIBO-positive patients $(\mathrm{P}<0.05)$. Following rifaximin treatment, 6 of the $18(33.33 \%)$ SIBO-positive patients had improved, as evaluated by GHBT and VAS. Additionally, the GISS in the SIBO-turned-negative group had improved significantly compared with that in the non-turned-negative group $(\mathrm{P}<0.05)$. The symptoms of all 18 SIBO-positive patients following rifaximin treatment improved notably, particularly diarrhea $(\mathrm{P}<0.05)$. In conclusion, postoperative CRC patients are more likely to develop SIBO compared with healthy individuals, and SIBO may aggravate digestive symptoms. The administration of rifaximin improved the overall gastrointestinal symptoms, particularly diarrhea, in SIBO-positive patients.
\end{abstract}

Correspondence to: Ms. Lin Xu, Department of Gastroenterology, Qingdao Municipal Hospital Affiliated to Qingdao University, 5 Donghai Middle Road, Qingdao, Shandong 266071, P.R. China E-mail: xulin1968@163.com

Abbreviations: CRC, colorectal cancer; FBH, fasting breath hydrogen; GHBT, glucose hydrogen breath test; GISS, gastrointestinal symptoms score; IBS, irritable bowel syndrome; SIBO, small intestinal bacterial overgrowth; VAS, visual analogue scale

Key words: colorectal cancer, gastrointestinal symptom score, hydrogen breath test, rifaximin, small intestinal bacterial overgrowth

\section{Introduction}

Healthy individuals are equipped with defense mechanisms against bacterial overgrowth and the small intestine is relatively sterile (1). When one of those mechanisms is impaired, small intestinal bacterial overgrowth (SIBO) may occur. SIBO is usually caused by abnormal intestinal structure, intestinal dysfunction and impaired intestinal mucosal function (2). SIBO is a serious condition associated with a number of diseases, which may severely affect further treatment of the patients and compromise their quality of life.

Colorectal cancer (CRC) is one of the most common gastrointestinal malignant tumors. With the improvement of living standards and changes in dietary habits, the incidence and mortality of CRC have been continuously increasing in recent years (3). Moreover, with the advances in endoscopy and medical technology, the resection rate of CRC patients has increased. Postoperative CRC patients are commonly affected by symptoms such as unformed stools, constipation, bloating and abdominalgia. However, remission may be achieved with anti-SIBO treatments, such as bifid triple viable capsule and compound lactobacillus acidophilus, which are administered to regulate the intestinal flora based on the original treatment.

CRC and SIBO may exhibit overlapping symptoms. Moreover, the postoperative changes in the anatomical structure of the intestinal tract provides SIBO with breeding grounds in postoperative $\mathrm{CRC}$ patients. Therefore, it is reasonable to hypothesize that SIBO is likely to occur in such patients. On the basis of this hypothesis, we investigated whether treatment for SIBO may improve the digestive symptoms of postoperative CRC patients.

\section{Patients and methods}

Patients. A total of 43 postoperative CRC patients, aged 38-83 years (26 men and 17 women), who were hospitalized at the departments of Gastroenterology, General Surgery, Hepatobiliary Surgery and Oncology of Qingdao Municipal Hospital between January, 2012 and January, 2014, were selected. The patients were diagnosed with CRC through imaging examinations such as gastroscopy, colonoscopy and barium meal for the entire digestive system, and other accessory examinations, such as measurement of tumor markers. 
The diagnoses were confirmed with histopathological examinations following surgical removal of the tumors. All the patients signed written informed consent forms for the examinations. Furthermore, 30 healthy individuals (16 men and 14 women), aged 24-62 years, were recruited from the outpatient clinic and schools for the control group.

All the investigated subjects were required to meet the following criteria: i) No other conditions, such as diabetes, thyroid diseases, intestinal pseudo-obstruction, irritable bowel syndrome (IBS), or any other disease that may lead to poor gastrointestinal motility, and no lactose intolerance; ii) no use of antibiotics, antacids or probiotics over the last month; iii) no history of treatment with hormones, antidepressants and opioids; no long-term history of heavy smoking; iv) no colonoscopy or enema within the last 4 weeks; v) no history of renal insufficiency or infection.

All the procedures were performed under consensus agreements and in accordance with the Ethics Review Committee of Qingdao Municipal Hospital.

Glucose hydrogen breath test (GHBT). All the investigated subjects underwent GHBT (model: HHBT-01; Shenzhen CNNC Headway Biotechnology Co., Ltd.). i) Sedative hypnotics, smoking, and the consumption of cooked wheat-containing foods, vegetables, fruits, bean products, dairy products and other foods rich in cellulose, were not allowed one day before the test; ii) rice was allowed as staple food, with meat and eggs as non-staple foods on the day before the test, avoiding overeating; iii) consumption of solid or liquid foods was not allowed after 8:00 pm, to ensure a comparatively low value of fasting breath hydrogen (FBH) on the morning of the test (12-h fasting); iv) smoking was prohibited one day before and throughout the test. Eating and drinking were also not allowed during the test, as were strenuous exercise and sleeping. All the investigated subjects were advised to brush their teeth carefully before the test and were kept in a sitting position.

Experimental methods. Using the disposable gas mouth blowpipe, the investigated subject exhaled slowly for as long as possible, with the flow rate being controlled at $250 \mathrm{ml} / \mathrm{min}$. When the first exhalation was completed, the patients continued exhaling after taking a breath, until the figures on the liquid-crystal display screen stopped rising, which required $\sim 70 \mathrm{sec}$. First, FBH was measured. Subsequently, $50-80$ g glucose plus $200-250 \mathrm{ml}$ warm boiled water was used as the substrate (the patients gargled after having the substrate in order to minimize the effect of oral bacteria on the test). The expiratory hydrogen concentration was measured every $20 \mathrm{~min}$ after taking the substrate for $2 \mathrm{~h}$ in total.

SIBO diagnostic criteria. SIBO was considered to be positive if the expiratory hydrogen concentration had increased by $>12 \mathrm{ppm}$ after taking the glucose substrate, and negative otherwise.

Treatment. Patients diagnosed as SIBO-positive were continuously treated with oral rifaximin $(1,200 \mathrm{mg} / \mathrm{day})$ for 10 days. GHBT was performed again at the end of the treatment, to investigate and analyze the changes in expiratory hydrogen concentration and gastrointestinal symptoms score (GISS).
GISS. The visual analogue scale (VAS) was applied to all patients to evaluate the symptoms of diarrhea, constipation, bloating, abdominalgia, poor appetite and fever. The total score of each item was defined as the overall GISS. The symptom scores were compared separately between SIBO-positive and SIBO-negative patients, SIBO-positive patients before and after treatment, and SIBO eradicated and not eradicated groups.

Statistical analysis. Statistical analysis of the results was conducted using SPSS v19.0 software (Armonk, NY, USA). Qualitative data were evaluated using the $\chi^{2}$ test and $\mathrm{P}<0.05$ was considered to indicate statistically significant differences. Two-sample t-tests for a difference in mean and the Mann-Whitney $U$ test of rank sum test were performed to check the normality and homogeneity of variance. $\mathrm{P}<0.05$ was considered to to indicate statistically significant differences.

\section{Results}

Prevalence of SIBO in postoperative CRC patients. Among the 43 postoperative patients with CRC, 18 were diagnosed as SIBO-positive. However, among the 30 healthy individuals in the control group, only 2 were found to be SIBO-positive (41.86 vs. $6.67 \% ; \mathrm{P}<0.05$ ). Comparing the $\mathrm{CRC}$ group with the control group, the age difference of the investigated subjects was statistically significant $(\mathrm{P}<0.05)$, while gender was not ( $\mathrm{P}>0.05)$ (Table I; Fig. 1).

Digestive tract symptoms of SIBO. As regards the incidence of abdominalgia (34.88\%), bloating (32.56\%), diarrhea (41.86\%), constipation (23.26\%), fever (9.30\%) and poor appetite (4.65\%) among the 43 patients, the incidence of diarrhea was the highest, followed by abdominalgia and bloating $(\mathrm{P}<0.05)$ (Table II).

Among the 18 SIBO-positive patients, the difference among symptoms was statistically significant $(\mathrm{P}<0.05)$. The incidence of diarrhea was the highest, and it was significantly higher compared with that among SIBO-negative patients $(66.67$ vs. $24.0 \%$, respectively; $\mathrm{P}<0.05)$. Among the 25 SIBO-negative patients, the difference among symptoms was statistically significant $(\mathrm{P}<0.05)$ and the incidence of abdominalgia was the highest, although the difference was not statistically significant ( 32.0 vs. $38.89 \%$; $\mathrm{P}>0.05)$. The differences among all the other symptoms were not statistically significant $(\mathrm{P}>0.05)$ (Table II; Fig. 2).

VAS was applied to all the patients to calculate GISS. The value of SIBO-positive patients was significantly higher compared with that of SIBO-negative patients (8 vs. 4, respectively; $\mathrm{P}<0.05)$. Among 6 gastrointestinal symptoms, every score in the SIBO-positive patients was higher compared with that in SIBO-negative patients, but only the difference in diarrhea was statistically significant (7 vs. 5, respectively; $\mathrm{P}<0.05$ ) (Table III).

Effect of rifaximin on SIBO. SIBO-positive patients were continuously treated with rifaximin $(1,200 \mathrm{mg} / \mathrm{day})$ for 10 days . GHBT was performed at the end of the treatment course and GISS was calculated. Following rifaximin treatment, the overall GISS was lower compared with that prior to treatment (8 vs. 4 , respectively; $\mathrm{P}<0.05$ ). Only the difference in diarrhea was statistically significant compared with the values prior to 
Table I. Characteristics of study subjects and prevalence of SIBO.

\begin{tabular}{lcccccc}
\hline & \multicolumn{3}{c}{ Groups } & & \multicolumn{2}{c}{ Prevalence } \\
\cline { 2 - 3 } Characteristics & $\begin{array}{c}\text { CRC } \\
(\mathrm{n}=43)\end{array}$ & $\begin{array}{c}\text { Control } \\
(\mathrm{n}=30)\end{array}$ & & P-value & $\begin{array}{c}\text { SIBO-positive } \\
(\mathrm{n}=18)\end{array}$ & $\begin{array}{c}\text { SIBO-negative } \\
(\mathrm{n}=25)\end{array}$ \\
\hline SIBO prevalence, $\%$ & 41.89 & 6.67 & 0.001 & - & - \\
Age, years \pm SD & $63.67 \pm 11.09$ & $41.30 \pm 13.84$ & & 0.000 & $68.72 \pm 10.15$ & $60.04 \pm 10.45$ \\
Male/female & $26 / 17$ & $16 / 15$ & & 0.544 & $11 / 7$ & 0.010 \\
\hline
\end{tabular}

SIBO, small intestinal bacterial overgrowth; CRC, colorectal cancer; SD, standard deviation.

Table II. Prevalence of gastrointestinal symptoms.

\begin{tabular}{|c|c|c|c|c|}
\hline \multirow{2}{*}{$\begin{array}{l}\text { Gastrointestinal } \\
\text { symptoms }\end{array}$} & \multirow[b]{2}{*}{ Overall prevalence $(\%)$} & \multicolumn{2}{|c|}{ Prevalence, \% (n) } & \multirow[b]{2}{*}{ P-value } \\
\hline & & SIBO-positive $(n=18)$ & SIBO-negative $(n=25)$ & \\
\hline Abdominalgia & 34.88 & $38.89(7)$ & $32.00(8)$ & 0.640 \\
\hline Bloating & 32.56 & $50.00(9)$ & $20.00(5)$ & 0.038 \\
\hline Diarrhea & 41.86 & $66.67(12)$ & $24.00(6)$ & 0.005 \\
\hline Constipation & 23.26 & $33.33(6)$ & $16.00(4)$ & 0.336 \\
\hline Fever & 9.30 & $16.67(3)$ & $4.00(1)$ & 0.380 \\
\hline Poor appetite & 4.65 & $11.11(2)$ & $0.0(0)$ & 0.092 \\
\hline
\end{tabular}

SIBO, small intestinal bacterial overgrowth.

Table III. GISS of SIBO-positive and SIBO-negative groups prior to treatment.

\begin{tabular}{lccr}
\hline & \multicolumn{2}{c}{ Median severity score (range) } \\
\cline { 2 - 3 } Gastrointestinal symptoms & SIBO-positive $(\mathrm{n}=18)$ & SIBO-negative $(\mathrm{n}=25)$ & \\
\hline Global GISS & $8(6-14.25)$ & $4(1-7)$ & 0.002 \\
Abdominalgia & $5(2-6)$ & $3(2-6)$ & 0.604 \\
Bloating & $6(3-7.5)$ & $4(2.5-5.5)$ & 0.249 \\
Diarrhea & $7(4-8)$ & $5(3-6.25)$ & 0.036 \\
Constipation & $5.5(2.75-6.5)$ & $6.5(4.5-7)$ & 0.386 \\
Fever & $6(4-6)$ & $4(4-4)$ & 0.317 \\
Poor appetite & $5.5(3.75-4.5)$ & $0(0-0)$ & 0.102
\end{tabular}

SIBO, small intestinal bacterial overgrowth; GISS, gastrointestinal symptoms score.

treatment ( 7 vs. 3 , respectively; $\mathrm{P}<0.05$ ), while no statistically significant differences were observed regarding the rest of the symptoms (Table IV).

In addition, 6 of the 18 SIBO-positive patients became SIBO-negative, with a negative conversion ratio of $33.33 \%$. No symptoms were experienced by the 2 SIBO-positive patients in the control group; thus, no treatment was administered (Table V).

\section{Discussion}

In recent years, several studies demonstrated that SIBO was correlated with several digestive system diseases or symptoms, such as IBS, cirrhosis and acute severe pancreatitis. Postoperative CRC patients also exhibited several digestive problems $(4,5)$. In this study, GHBT was performed in 43 postoperative patients with $\mathrm{CRC}$ and 30 healthy individuals. A total of $41.86 \%$ postoperative CRC patients were found to have an abnormal expiratory hydrogen concentration, while $6.67 \%$ of the control group were found to be SIBO-positive. The overall symptoms of the CRC patients with SIBO, particularly diarrhea, were improved following rifaximin treatment, whereas $33.33 \%$ of the SIBO-positive patients became SIBO-negative. Therefore, SIBO detection and treatment were necessary for postoperative CRC patients. 
Table IV. GISS of the SIBO-positive group before and after treatment.

Median severity score (range)

Gastrointestinal symptoms

\begin{tabular}{lccr} 
Gastrointestinal symptoms & Pre-treatment & Post-treatment & P-value \\
\hline Global GISS & $8(6-14.25)$ & $4(3-9.50)$ & 0.037 \\
Abdominalgia & $5(2-6)$ & $3(2-6)$ & 0.561 \\
Bloating & $6(3-7.50)$ & $3(1.50-5.50)$ & 0.120 \\
Diarrhea & $7(4-8)$ & $3(2-4)$ & 0.008 \\
Constipation & $5.5(2.75-6.50)$ & $4(2.50-5.80)$ & 0.450 \\
Fever & $6(4-6)$ & $3(3-3)$ & 0.102 \\
Poor appetite & $5.5(3.75-4.50)$ & $6(3-6)$ & 0.655 \\
\hline
\end{tabular}

SIBO, small intestinal bacterial overgrowth; GISS, gastrointestinal symptoms score.

Table V. Comparison between SIBO turned-negative group and SIBO non-turned-negative group.

\begin{tabular}{lccc}
\hline Global GISS & Pre-treatment & P-value & Post-treatment \\
\hline Turned-negative group & $8(6-10.80)$ & 0.742 & $3(1-4)$ \\
Non-turned-negative group & $7.5(6-26.3)$ & & $7.5(3.3-20.8)$ \\
\hline
\end{tabular}

Values are presented as median (range). SIBO, small intestinal bacterial overgrowth; GISS, gastrointestinal symptoms score.

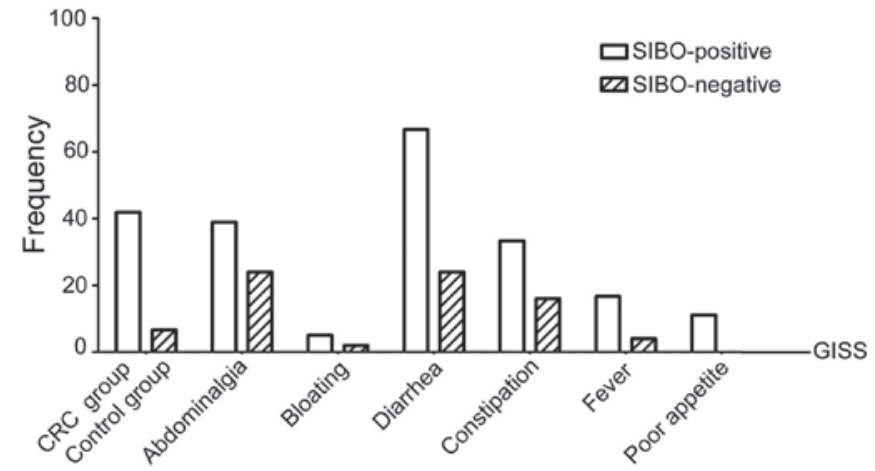

Figure 1. Prevalence of gastrointestinal symptoms. SIBO, small intestinal bacterial overgrowth; GISS, gastrointestinal symptoms score; CRC, colorectal cancer.

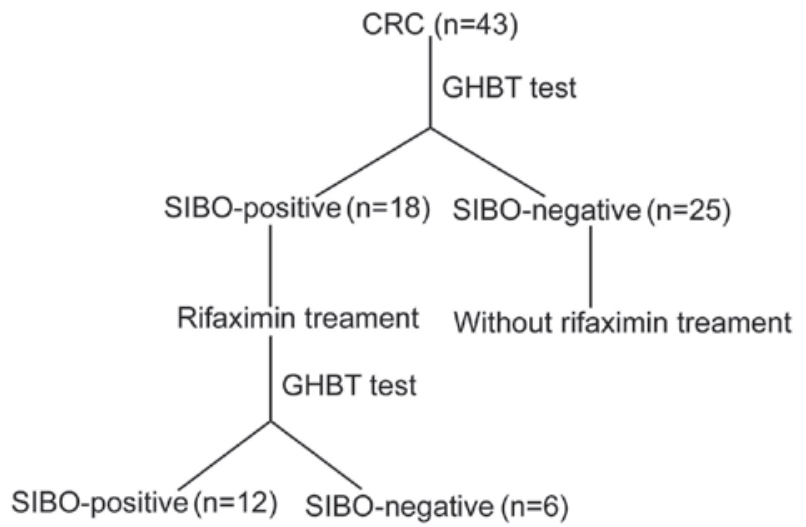

Figure 2. Flow chart of the study process. CRC, colorectal cancer; SIBO, small intestinal bacterial overgrowth; GHBT, glucose hydrogen breath test.
It has been demonstrated that SIBO, as well as bacteremia, sepsis and intestinal endotoxemia induced by SIBO, are serious conditions associated with a number of diseases, which may affect further treatment of the patients and severely compromise their quality of life. The overgrown bacteria compete with the host for dietary vitamin B12, interfere with the metabolism of bile salts and affect the absorption of amino acids, thereby leading to B12 hypovitaminosis, diarrhea and hypoalbuminemia (6). Furthermore, the overgrown small intestinal bacteria may greatly increase the number of the bacteria that adhere to the intestinal wall, subsequently producing a large number of metabolites and toxins that may damage the structure of the intestinal mucosa $(7,8)$. Therefore, SIBO adversely affects the intestinal tract through various factors, thereby aggravating gastrointestinal tract symptoms.

It was found that the clinical symptoms of the patients with SIBO were more severe compared with those of patients without SIBO. In addition, among all SIBO-positive patients, the incidence of diarrhea was the highest, followed by abdominalgia and bloating. However, among SIBO-negative patients, the incidence of abdominalgia was the highest, while that of diarrhea and bloating was not as high. This difference in diarrhea scores was statistically significant $(\mathrm{P}<0.05)$. The points mentioned above indicate that patients with severe gastrointestinal symptoms are more likely to have SIBO, particularly when diarrhea is the main symptom. SIBO may aggravate the patients' gastrointestinal symptoms, and treating SIBO improves such symptoms.

The symptoms of IBS may be significantly alleviated through the application of probiotics or antibiotics to eradicate SIBO. Previously reported data even demonstrated that the gastrointestinal symptoms of some patients with IBS were completely relieved following eradication of SIBO (9-12). 
Rifaximin is a semi-synthetic antibiotic designed based on rifamycin. It is a type of broad-spectrum oral bactericidal that cannot be absorbed through the intestinal wall. Thus, there are no known interactions with other drugs and its adverse reactions are mild $(13,14)$. Recently, an increasing number of researchers verified the therapeutic effect of rifaximin on intestinal diseases. A dose of $550 \mathrm{mg}$ rifaximin three times a day for 14 days has been used to treat patients with IBS, as reported by Pimentel et al (15); they found that $40.8 \%$ of the symptoms improved, compared with $31.2 \%$ of IBS patients treated with placebo $(\mathrm{P}<0.05)$. In another study, IBS patients treated with rifaximin for 14 days were followed up and it was found that their symptoms of bloating, diarrhea and abdominalgia were significantly improved within 3 months (16). The study of Biancone et al (17) demonstrated that short-course treatment with rifaximin for patients with Crohn's disease combined with SIBO was effective. All those studies indicate that rifaximin is effective against SIBO.

In our study, it was found that, after treatment with rifaximin for 10 days, the GISS of 18 SIBO-positive patients decreased $(\mathrm{P}<0.05)$, and $33.33 \%$ of those were no longer SIBO-positive. The overall symptoms, particularly diarrhea, were significantly alleviated following treatment for SIBO, proving that treatment for SIBO is an effective measure for alleviating the gastrointestinal symptoms of postoperative patients with CRC. Therefore, the identification and timely treatment of SIBO are crucial.

Although treatment with rifaximin for SIBO may relieve some of the symptoms, it did not relieve all the gastrointestinal symptoms, and the turned-negative rate was rather low. Therefore, more effective agents for the eradication of SIBO are urgently required.

\section{Acknowledgements}

The present study was supported in part by a grant from the Qingdao Science and Technology Bureau (grant no. 13-1-3-14-NSH to Ms. Lin Xu).

\section{References}

1. Bustillo I, Larson H and Saif MW: Small intestine bacterial overgrowth: An underdiagnosed cause of diarrhea in patients with pancreatic cancer. JOP 10: 576-578, 2009.
2. Bures J, Cyrany J, Kohoutova D, Förstl M, Rejchrt S, Kvetina J, Vorisek V and Kopacova M: Small intestinal bacterial overgrowth syndrome. World J Gastroenterol 16: 2978-2990, 2010.

3. Durko L and Malecka-Panas E: Lifestyle modifications and colorectal cancer. Curr Colorectal Cancer Rep 10: 45-54, 2014.

4. Madrid AM, Hurtado C, Gatica S, Chacón I, Toyos A and Defilippi C: Endogenous ethanol production in patients with liver cirrhosis, motor alteration and bacterial overgrowth. Rev Med Chil 130: 1329-1334, 2002 (In Spanish).

5. Fisher D, Rajon D, Breitz H, Goris M, Bolch W and Knox S: Dosimetry model for radioactivity localized to intestinal mucosa. Cancer Biother Radiopharm 19: 293-307, 2004.

6. Fan X and Sellin JH: Review article: Small intestinal bacterial overgrowth, bile acid malabsorption and gluten intolerance as possible causes of chronic watery diarrhoea. Aliment Pharmacol Ther 29: 1069-1077, 2009.

7. Husebye E, Hellström PM, Sundler F, Chen J and Midtvedt T: Influence of microbial species on small intestinal myoelectric activity and transit in germ-free rats. Am J Physiol Gastrointest Liver Physiol 280: G368-G380, 2001.

8. Passos MC, Serra J, AzpirozF, Tremolaterra F and Malagelada JR: Impaired reflex control of intestinal gas transit in patients with abdominal bloating. Gut 54: 344-348, 2005.

9. Basseri RJ, Weitsman S, Barlow GM and Pimentel M: Antibiotics for the treatment of irritable bowel syndrome. Gastroenterol Hepatol (NY) 7: 455-493, 2011.

10. Jolley J: High-dose rifaximin treatment alleviates global symptoms of irritable bowel syndrome. Clin Exp Gastroenterol 4: 43-48, 2011.

11. Marie I, Ducrotté P, Denis P, Menard JF and Levesque H: Small intestinal bacterial overgrowth in systemic sclerosis. Rheumatology (Oxford) 48: 1314-1319, 2009.

12. Tan AH, Mahadeva S, Thalha AM, Gibson PR, Kiew CK, Yeat CM, Ng SW, Ang SP, Chow SK, Tan CT, et al: Small intestinal bacterial overgrowth in Parkinson's disease. Parkinsonism Relat Disord 20: 535-540, 2014.

13. Saadi $M$ and McCallum RW: Rifaximin in irritable bowel syndrome: Rationale, evidence and clinical use. Ther Adv Chronic Dis 4: 71-75, 2013.

14. Karanje RV, Bhavsar YV, Jahagirdar KH and Bhise KS: Formulation and development of extended-release micro particulate drug delivery system of solubilized rifaximin. AAPS PharmSciTech 14: 639-648, 2013.

15. Pimentel M, Morales W, Chua K, Barlow G, Weitsman S, Kim G, Amichai MM, Pokkunuri V, Rook E, Mathur R and Marsh Z: Effects of rifaximin treatment and retreatment in nonconstipated IBS subjects. Dig Dis Sci 56: 2067-2072, 2011.

16. Meyrat P, Safroneeva E and Schoepfer AM: Rifaximin treatment for the irritable bowel syndrome with a positive lactulose hydrogen breath test improves symptoms for at least 3 months. Aliment Pharmacol Ther 36: 1084-1093, 2012.

17. Biancone L, Vernia P, Agostini D, Ferrieri A and Pallone F: Effect of rifaximin on intestinal bacterial overgrowth in Crohn's disease as assessed by the $\mathrm{H}_{2}$-glucose breath test. Curr Med Res Opin 16: 14-20, 2000. 